\title{
Silver-nanoparticle-decorated carbon nanoscaffolds: Application as a sensing platform
}

\author{
S. J. Henley, ${ }^{\text {a) }}$ J. D. Carey, and S. R. P. Silva \\ Nano-Electronics Centre, Advanced Technology Institute, School of Electronics and Physical Sciences, \\ University of Surrey, Guildford, GU2 7XH, United Kingdom
}

(Received 18 July 2006; accepted 20 September 2006; published online 3 November 2006)

\begin{abstract}
Cluster-assembled nanostructured carbon with a fractal morphology is employed as a large-area surface scaffold for metal decoration. By depositing silver by pulsed laser ablation densely packed, distributions of metal nanoparticles are produced. The authors show, using the surface-enhanced Raman effect and the modification of fluorescence quantum yields near metallic surfaces, that silver-coated nanostructured carbon can be used to sense low concentrations of biomolecules.
\end{abstract}

(C) 2006 American Institute of Physics. [DOI: 10.1063/1.2378431]

Trace-level optical detection finds a wide range of applications in security screening, health care, and environmental monitoring. However, one major hurdle for such applications is the relatively weak optical signal from most molecules. One solution to this problem is surface-enhanced Raman spectroscopy (SERS), ${ }^{1-4}$ using the enhancement of the scattered intensity produced by nanostructured metal surfaces (typically $\mathrm{Ag}, \mathrm{Au}$, or $\mathrm{Cu}$ ), which can increase the sensitivity by orders of magnitude. The magnitude of the enhancement is strongly affected by the precise nanostructure; thus research is ongoing to produce reliable, repeatable nanostructured surfaces. 5

Pulsed laser ablation ${ }^{6}$ (PLA) of graphite results in the deposition of different materials depending on the pressure of gas present in the growth chamber. ${ }^{7,8}$ Growth in inert gas yields low density nanostructured amorphous carbon (NAC) films, ${ }^{8,9}$ which can exhibit a range of useful properties, including high porosities. ${ }^{10}$ Recent work on high-repetitionrate PLA of graphite in high background pressures of argon has shown that these conditions deposit carbon nanofoam. ${ }^{11}$ This material has an open, fractal-like, morphology which presents a large surface area. ${ }^{11}$ Here, we investigate the decoration of these NAC materials with $\mathrm{Ag}$ nanoparticles. By coupling the large surface area of these self-assembled carbon nanoscaffolds with a SERS-active component efficient, low-cost sensor platforms may be realized. These largesurface-area materials may have applications as a capture medium for airborne analytes in security screening. Similar large-area SERS substrates have been produced by silvercoating porous silicon substrates. ${ }^{12}$

The initial carbon films were deposited by PLA of a 99.99\% pure graphite target using a Lambda Physik LPX $210 \mathrm{i}$ excimer laser operating at $248 \mathrm{~nm}$, with a pulse duration of $25 \mathrm{~ns}$. $60 \mathrm{~mJ}$ laser pulses were focused onto the rotating target, producing an incident fluence of $\sim 6 \mathrm{~J} / \mathrm{cm}^{2}$. The growth chamber was evacuated using a turbomolecular pump to $10^{-5} \mathrm{~Pa}$, then backfilled with argon to the required pressure. Films were deposited onto $\mathrm{Si}$ and Corning glass substrates at a background argon pressure $\left(P_{\mathrm{Ar}}\right)$ of $45 \mathrm{~Pa}$ using 6000 laser shots. A more detailed discussion of the effect of $P_{\text {Ar }}$ on the morphology of the carbon films can be found elsewhere. ${ }^{8}$ The metal coatings were also deposited

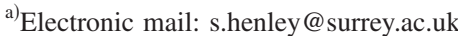

using the same laser system, at a higher fluence of $10 \mathrm{~J} / \mathrm{cm}^{2}$, without the presence of Ar. The nanostructure of the films was investigated using a Philips CM200 transmission electron microscope (TEM). The SERS activity of the films was investigated using a Renishaw Ramanscope, using $488 \mathrm{~nm}$ excitation and a solution of Rhodamine $6 \mathrm{G}(\mathrm{R} 6 \mathrm{G}, \sim 1 \mathrm{mM})$ was used as the analyte. One drop of the solution was applied to the surface of the film and allowed to dry. In addition to SERS, the enhancement of the fluorescence from the protein bovine serum albumin labeled with fluorescein isothiocyanate (BSA-FITC) was also measured, after the samples had been dipped into a solution containing the protein at $1 \mathrm{mg} / \mathrm{ml}$, and then washed in distilled water.

TEM images of the initial NAC film are shown in Figs. 1(a) and 1(b). The nanostructure consists of nanoscale clusters with diameters of $\sim 10 \mathrm{~nm}$, which are assembled in a fractal-like arrangement suggesting that the growth mechanism is characterized by diffusion-limited aggregation. ${ }^{13}$ After deposition of the NAC scaffold, we investigated the surface decoration of this material with silver nanoparticles. Figures 1(c)-1(f) show a series of TEM images of NAC films coated with different amounts of $\mathrm{Ag}$, equivalent to metal film thicknesses of $1,3,5$, and $15 \mathrm{~nm}$ if the Ag had been deposited onto a bare substrate. From this figure, it can be observed that the Ag does not uniformly coat the carbon surface but forms discrete nanoparticles, which increase in size and coalesce as the amount of silver is increased. By $15 \mathrm{~nm}$ of $\mathrm{Ag}$, the metal particles have become quite large and the coating have approached continuity. Figure 2 shows histograms of the nanoparticle size for equivalent Ag film thicknesses of (a) 1, (b) 3, and (c) $5 \mathrm{~nm}$. These distributions were obtained from TEM images using commercial image analysis software (SCION IMAGE). The distributions are not monodisperse and have a broad tail to a larger particle size, which becomes more significant as the amount of silver deposited is increased. Figure 2(d) shows a plot of mean particle size as a function of equivalent Ag film thickness. The optimum particle size for SERS is difficult to determine, as the observed enhancement is affected by many factors including the interparticle spacing in aggregates and the dielectric properties of the surrounding medium. However, theoretically, the maximum enhancement for a single particle should be in the size range of $10-100 \mathrm{~nm}^{3}$. For smaller particles, electron scattering at the surfaces becomes dominant and the quality of the 


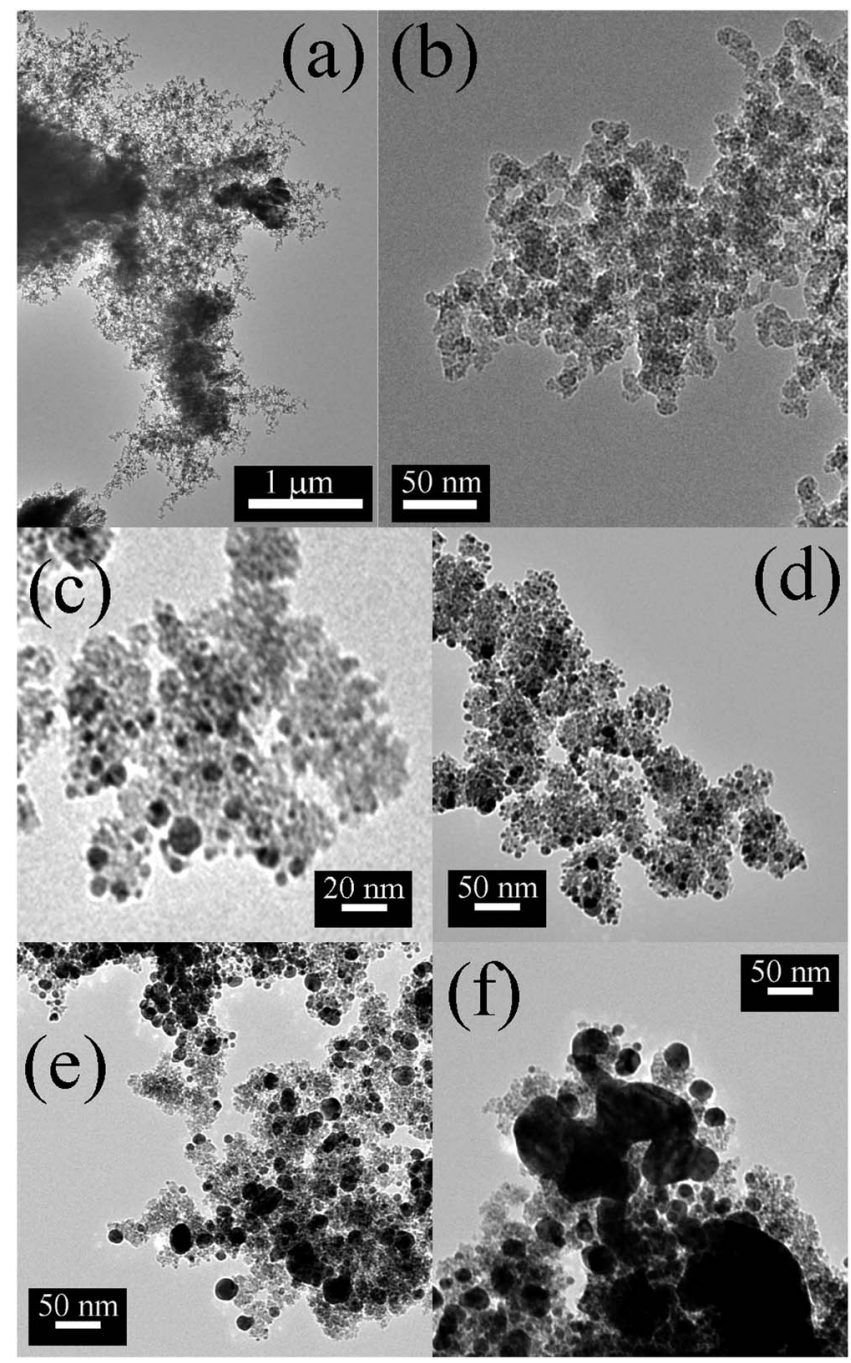

FIG. 1. [(a)-(b)] TEM images of the initial NAC film, deposited at $P_{\mathrm{Ar}}$ $=45 \mathrm{~Pa}$, showing (a) the fractal morphology and (b) the nanoscale clusters. [(c)-(f)] Series of TEM images of NAC films coated with different amounts of $\mathrm{Ag}$, equivalent to metal film thicknesses of (c) 1, (d) 3, (e) 5, and (f) $15 \mathrm{~nm}$.

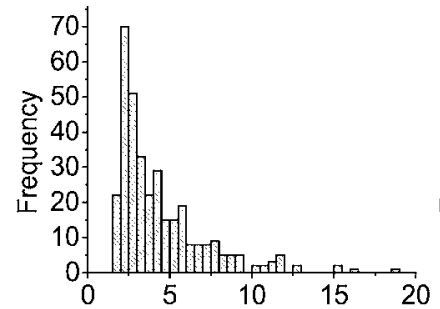

(a)
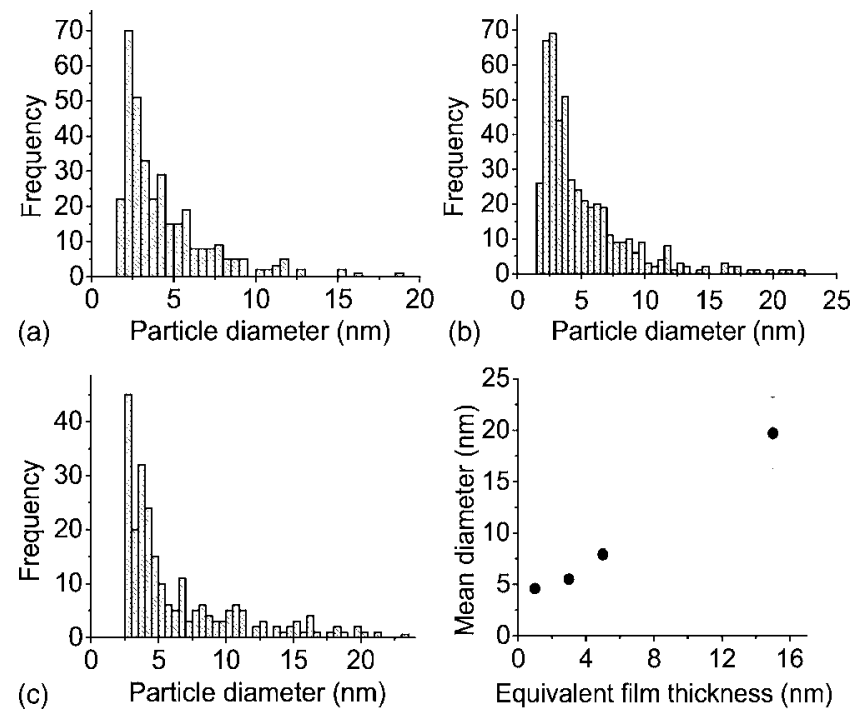

(b)

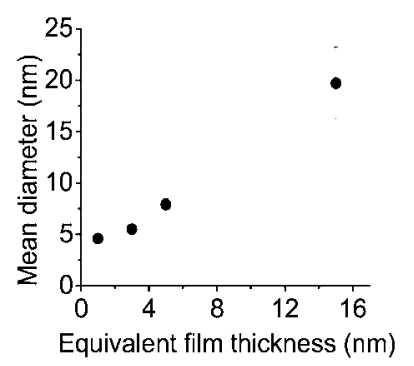

FIG. 2. Histograms of nanoparticle size for equivalent $\mathrm{Ag}$ film thicknesses of (a) 1, (b) 3, and (c) $5 \mathrm{~nm}$. (d) Shows a plot of mean particle size as a function of equivalent Ag film thickness.

Downloaded 30 Mar 2009 to 131.227.178.132. Redistribution subject to AlP license or copyright; see http://apl.aip.org/apl/copyright.jsp
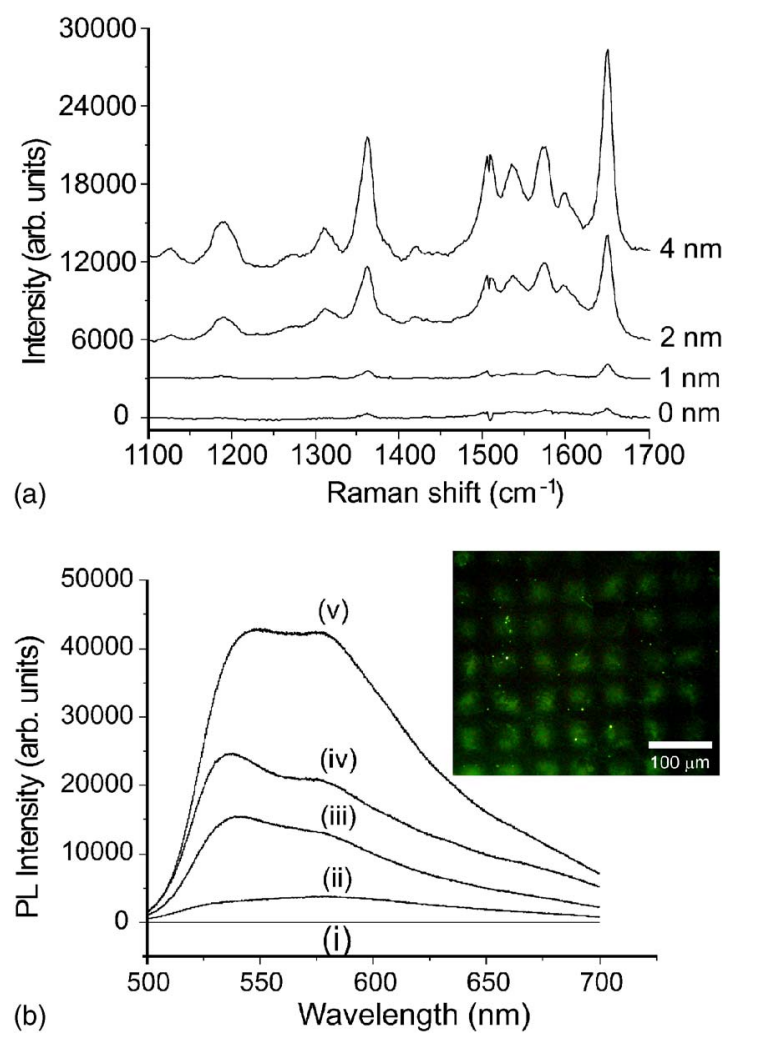

FIG. 3. (Color online) (a) Raman spectra from drops of the R6G solution on NAC substrates with Ag coatings of $0,1,2$, and $4 \mathrm{~nm}$. A linear photoluminescence background has been subtracted, and the spectra have been offset for clarity. (b) Florescence spectra from BSA-FTIC on a series of different substrates. (i) Flat amorphous carbon, (ii) untreated NAC, (iii) NAC+an equivalent $2 \mathrm{~nm} \mathrm{Ag}$ coating, (iv) NAC $+6 \mathrm{~nm} \mathrm{Ag}$, and (v) NAC+4 nm Ag. Inset: Fluorescence image of a protein coated array of NAC patches, patterned onto a flat amorphous carbon surface.

resonance is reduced, and with it the SERS enhancement. ${ }^{3}$ Here, we would expect SERS enhancement to significantly increase with increasing $\mathrm{Ag}$ deposition, as a larger fraction of the nanoparticles will be within this size range.

Before studying the use of these substrates for SERS, it is important to investigate the optical properties of this composite material to eliminate background effects. The optical absorption spectra were measured from a series of NAC samples on a glass, coated with $\mathrm{Ag}$ (not shown here). A pronounced optical absorption band around $400 \mathrm{~nm}$, which is due to the surface plasmon resonance of Ag nanoparticles, ${ }^{14}$ was observed. Before testing the SERS enhancement from an added analyte, the Raman spectrum of the NAC scaffold was also measured, as this will also be enhanced, and may have to be subtracted. An enhancement in the $D$ and $G$ Raman peaks, characteristic of amorphous carbon, ${ }^{15}$ was observed. However, these peaks were broad and can be readily subtracted from the total signal. Figure 3(a) shows Raman spectra from a drop of R6G solution placed onto a series of $\mathrm{Ag}$ decorated films. R6G evidences a series of sharp Raman features in this energy range and is typically used as analyte for testing the surface-enhanced Raman effect. A strong enhancement of the Raman signal from the R6G can be observed clearly along with an improved signal-to-noise ratio. For the $4 \mathrm{~nm} \mathrm{Ag}$ coating, the contribution to the Raman signal from the carbon scaffold is small compared to the signal from the R6G; hence for this analyte no correction would be required. 
In addition to SERS, silver nanoparticles can also affect the fluorescence from molecules emitting in their vicinity, ${ }^{16}$ which is attributed to interactions of the excited-state fluorphores with free electrons in the metal, modifying the radiative decay rate. To investigate this effect here, the Ag coated NAC sample were dipped into a solution containing the fluorescent-tagged protein BSA-FTIC and then washed in distilled water. Figure 3(b) shows the fluorescence spectrum from the protein on a series of different substrates. Initially, we measured the fluorescence from a flat amorphous carbon surface, deposited by PLA in vacuum, which had been dipped in the protein solution. After washing, no fluorescence could be detected from this surface [curve (i), Fig. 3(b))] Subsequently, an untreated NAC sample was dipped into the same protein solution. After washing, fluorescence could be detected [curve (ii), Fig. 3(b)], demonstrating that the protein attaches readily to the NAC surface and remains after washing. To demonstrate this further, an array of NAC patches was patterned onto a flat amorphous carbon surface by depositing through a mask. After treating with the protein solution and washing, the sample was imaged in a fluorescence microscope [see the inset of Fig. 3(b)]. The NAC areas appear bright in this image, whereas the flat carbon surface produces little fluorescence. This indicates that the protein attaches to the NAC, allowing significant amounts to be concentrated in a small area. To examine the surface enhancement of the fluorescence, Ag functionalized NAC samples were exposed to the protein solution. The fluorescence from these surfaces was strongly enhanced by the presence of $\mathrm{Ag}$ nanoparticles [curves (iii)-(v), Fig. 3(b)]. However, the enhancement was less for the $6 \mathrm{~nm} \mathrm{Ag}$ coating compared with the $4 \mathrm{~nm}$ coating. This can be explained by the observation that, for the thicker Ag coating, the surface became hydrophobic, and the protein solution would not wet the surface.

In conclusion, by depositing silver onto large-surfacearea carbon scaffolds, densely packed, three-dimensional distributions of metal nanoparticles were produced. We investigated this silver-coated carbon nanofoam as a possible sensor platform and demonstrated strong enhancement of the Ra- man and fluorescence from analytes on these substrates. Although the SERS enhancement observed was less than that typically seen with flat colloidal silver films, ${ }^{17}$ these materials have the advantage of a large-surface-area, threedimensional nanostructure, which may allow novel applications in security screening to capture airborne analytes. We also demonstrated, using fluorescent spectroscopy, that biomolecules, such as proteins, can attach to the carbon scaffold, suggesting possible biosensor applications.

The authors thank the EPSRC Portfolio Grant for funding. One of the authors (S.J.H.) is grateful to J. M. Hayes, J. Filik, G. M. Fuge, and P. W. May from the University of Bristol for assistance with the Raman/PL and to C. Jeynes for the fluorescence imaging.

${ }^{1}$ Y. Lu, G. L. Liu, and L. P. Lee, Nano Lett. 5, 5 (2005).

${ }^{2}$ A. Campion and P. Kambhampati, Chem. Soc. Rev. 27, 241 (1998).

${ }^{3}$ M. Moskovits, J. Raman Spectrosc. 36, 485 (2005).

${ }^{4}$ S. J. Henley, J. D. Carey, and S. R. P. Silva, Appl. Phys. Lett. 88, 081904 (2006).

${ }^{5}$ N. M. B. Perney, J. J. Baumberg, M. E. Zoorob, M. D. B. Charlton, S. Mahnkopf, and C. M. Netti, Opt. Express 14, 847 (2006).

${ }^{6}$ M. N. R. Ashfold, F. Claeyssens, G. Fuge, and S. J. Henley, Chem. Soc. Rev. 33, 23 (2004).

${ }^{7}$ R. K. Thareja and R. K. Dwivedi, Phys. Lett. A 222, 199 (1996).

${ }^{8}$ S. J. Henley, J. D. Carey, S. R. P. Silva, G. M. Fuge, M. N. R. Ashfold, and D. Anglos, Phys. Rev. B 72, 205413 (2005).

${ }^{9}$ Z. Geretovszky, T. Haraszti, T. Szörényi, F. Antoni, and E. Fogarassy, Appl. Surf. Sci. 208-209, 566 (2003).

${ }^{10}$ M. P. Siegal, D. L. Overmyer, R. J. Kottenstette, D. R. Tallant, and W. G. Yelton, Appl. Phys. Lett. 80, 3940 (2002).

${ }^{11}$ A. V. Rode, E. G. Gamaly, and B. Luther-Davies, Appl. Phys. A: Mater. Sci. Process. 70, 135 (2000).

${ }^{12}$ S. Chan, S. Kwon, T. Koo, L. P. Lee, and A. A. Berlin, Adv. Mater. (Weinheim, Ger.) 15, 1595 (2003).

${ }^{13}$ T. A. Witten and L. M. Sander, Phys. Rev. Lett. 47, 1400 (1981).

${ }^{14}$ W. P. Halperin, Rev. Mod. Phys. 58, 533 (1986).

${ }^{15}$ A. C. Ferrari and J. Robertson, Phys. Rev. B 61, 14095 (2000).

${ }^{16}$ J. R. Lakowicz, Y. Shen, S. D'Auria, J. Malicka, J. Fang, Z. Gryczynski, and I. Gryczynski, Anal. Biochem. 301, 261 (2002).

${ }^{17}$ P. C. Lee and D. J. Meisel, J. Phys. Chem. 86, 3391 (1982). 